\title{
Drug Delivery Research for the Future: Expanding the Nano Horizons and Beyond
}

Advances in drug delivery technologies have introduced numerous formulations and devices for improving drug efficacy and patient compliance. Such advances occurred as a result of contributions from scientists with diverse backgrounds and research interests. A wide range of drugdelivery products including oral, transdermal, and injectable depot formulations, among others, have entered the market and have helped millions of patients. The impact created by these diverse products has further fueled R\&D on numerous aspects of drug delivery in academia and industry. Research and review articles in the Journal of Controlled Release (JCR) have represented this diversity quite well since its launch in 1984 . Over the last decade, we, the editors of the JCR have noticed a clear shift from this diverse historical base to a strong focus on nanotechnology-based research. We would like to take this opportunity to reflect on the current trends in drug delivery research and provide guidelines for future research.

\section{Unprecedented attention to nanomedicine in oncology}

Over the last two decades, the drug delivery field has witnessed an explosive growth of research based on the use of nanotechnology for medical applications, broadly labeled as nanomedicine. Federal and private funding for nanomedicine research has led to numerous publications, start-ups, and an elevated public awareness of nanotechnology. This wave of excitement has positively impacted the field of drug delivery by channeling the imagination of researchers including pharmaceutical scientists, chemists, material scientists, engineers, physicists, biologists, and clinicians to nanomedicine. Collectively, this expansion has brought new tools and technologies to the drug delivery field.

Scientific advances in nanomedicine have laid the foundation for technologies that are geared for clinical translation. Start-ups and big pharmaceutical companies showed a marked increase in commercial interest and capital followed. Getting an approval of a new drug by the Food and Drug Administration (FDA) is an expensive and long process. The road to approved nanotechnology products is also long and tortuous. For the last 20 years, on average, 30 new drugs were approved by the U.S. FDA each year, and only a few nanomedicine-based drugs can be found on the approved list.

\section{Challenges facing the current nanomedicine for oncology articles}

The JCR editors applaud scientific advances in nanotechnology and clinical trials of nanomedicine formulations. At the same time, we take this opportunity to reflect on the current status and challenges that will serve as a guide into the future. It is not uncommon to see a JCR issue packed with articles on nanomedicine. An unusually high proportion of articles deal with various forms of nanoparticles for tumor targeting. We are at a point where the word 'nanomedicine' is often implicitly used to represent tumor-targeted drug delivery by nanoparticles. Such extreme focus on one aspect of a disease is quite uncharacteristic in the field of drug delivery that deals with a large number of formulations for various diseases.

\subsection{Research on nanoparticle-based tumor targeting follows a predictable pattern}

Most articles on tumor-targeted drug delivery by nanoparticle formulations follow a predictable pattern of organization: nanoparticle synthesis, in vitro cell-culture screening for efficacy, and in vivo mouse xenograft studies. The result of the studies is also predictable in that the nanoparticle formulations reduce the tumor size more than the control formulation does. This relative improvement, while valid within the scope of a specific study, may not represent the true advance necessary for a potential clinical benefit. It is imperative that oncology-focused nanomedicine research transcend this repetitive pattern for the betterment of the field, especially since the mouse xenograft model has failed to represent tumors in humans. Historically, advanced drug delivery technologies, in particular oral and transdermal sustained release formulations, have undergone successful clinical translation on the back of preclinical models that provide meaningful prediction of efficacy in humans. Hence, new pre-clinical test methods that better represent the human tumor environment must be developed. Until then, researchers should hold the rodent data to a very high standard so that the likelihood of its clinical impact is increased, even if the human data turns out to show significantly less efficacy than the mouse data suggests.

\subsection{Many disease targets in addition to cancer}

There is no doubt that cancer is one of the most formidable diseases in human health, but by no means the only one. Cardiovascular diseases, diabetes, CNS diseases, respiratory diseases, ocular diseases, and infections, among others, are serious health challenges that may benefit from nanotechnology. To date, however, the vast majority of nanomedicine articles focus on tumor-targeted nanoparticle formulations. The field will benefit significantly from increased attention on other diseases besides cancer.

\subsection{The nanoformulation-first approach}

When a disease is to be treated, sufficient attention must be paid first to its underlying biology and then a proper drug delivery system is developed. However, the approach taken in many articles submitted to the JCR is the reverse. This issue may be especially important with nanoparticlebased delivery systems intended for intravenous administration, yet we too often see articles where extraordinary emphasis has been placed on 
formulation first, and the disease to be treated is not always carefully considered. This formulation-first approach has stimulated significant creative thinking in the field and has produced thousands of nanoparticles with unique properties. The downside of this approach, however, is inadequate attention to human physiology and underlying disease biology, which often limits clinical translation. For example, most nanoparticles in the literature for tumor-targeted drug delivery rely on the enhanced permeability and retention (EPR) effect to approach tumors without studying the pathophysiology of tumors. A more practical and useful approach would be to keep the focus on the disease biology, allowing development of the right drug delivery systems, whether nanotechnologybased or not, to treat the disease. This disease-first approach is likely to yield more translatable solutions, nanoformulations included.

\section{Types of nanomedicine manuscripts expected in JCR}

Going forward, we encourage submission of manuscripts that diversify applications of nanotechnology to a wider range of diseases. We believe that such diversification will increase the likelihood of future success of nanotechnology by opening parallel channels for potential clinical impact. Rather than forcing a nanotechnology-based solution on a given disease, drug delivery scientists should test a diverse set of delivery approaches to arrive at optimal formulations that are clinically effective for many diseases.

\subsection{Manuscripts using proper controls for mouse xenograft studies}

Tumor-targeted drug delivery by nanoparticle formulations in mouse xenograft models has not always been predictive of clinical outcome in humans. This discrepancy originates from a number of factors, including the non-natural nature of tumor xenografts, high tumor-tobody mass ratio, and differences in vasculature. These issues are now well recognized and it is hoped that more sophisticated models will be developed soon. Researchers should be aware of these issues and make an effort to increase the rigor in their experimental models. In addition, the control formulation used in xenograft mouse studies is often a simple drug solution. In clinical studies, however, the test formulation is compared with clinical standard of care. Early incorporation of this clinical standard in pre-clinical studies and in appropriate animal models will assist in assessing potential clinical impact at an early stage.

\subsection{Manuscripts challenging current dogmas}

The JCR editors will continue to welcome manuscripts that report on nanoparticles for tumor targeting, but encourage the focus of such manuscripts to be on critical challenges in the field. Fundamental understanding of the interface of tumor physiology and nanoparticles is still critically lacking. Many manuscripts claim improved efficacy of tumor targeting by nanoparticle formulations in xenograft mouse models based on the EPR effect and affinity interactions in comparison with a control solution drug formulation. Yet the fundamental premise of the EPR effect in human tumors and affinity-based tumor targeting of nanoparticles remains elusive. We welcome manuscripts that put these assumptions to the test. Relevance of mouse models to human tumors is also a key issue in the field. We need to develop clinical solutions based on data, and not on assumptions. The relative tumor burden and growth rate in mice are unusually large as compared with that in human patients. Not surprisingly, positive results in xenograft tumors in mice treated with nanoparticles do not necessarily translate to humans. The presence of an active immune system, which exists in humans but not in some mouse models, also creates disparity between these models. As problematic as they might be, we realize that mouse models will remain the primary testbed for research for many reasons, including cost and logistics. However, we encourage authors to discuss potential challenges in translating mouse data to humans and alternative models that recapitulate tumor in humans better than the current mouse xenograft model.

\subsection{Manuscripts dealing with fundamental mechanisms and clinical} applications

We also welcome manuscripts that cover fundamental contributions to the field of nanomedicine beyond tumor-targeted drug delivery by intravenous administration. As discussed before, there are many other equally important applications of nanomedicine, including ocular, pulmonary, and oral delivery. Retrospective analysis of the outcomes of clinical trials and marketed products for deeper understanding of success, or lack thereof despite promising results in preclinical models, will be critical for future design and trials. Manuscripts describing the lack of efficacy with carefully designed experimental studies using appropriate testbeds are welcome. Contributions addressing challenges associated with characterization and scale-up manufacturing of nanoparticles, especially those that are applicable to a wide variety of nanoparticles, are also welcome. Nanotechnology promises to yield new revolutionary formulations that go beyond simple drug formulations. To realize this potential, we need to put such technologies to rigorous tests, critically evaluate the outcome and learn from both the positive and negative results. A large number of articles on nanoparticles do not sufficiently address this question and often fail to expertly assess the clinical or fundamental problems that may be solved. It is hoped that national funding agencies will continue to provide the resources to advance research in this area. It is in our best interest, however, to focus on key issues and ask, "What aspects of nanotechnology are key to future drug delivery systems, what are the key hurdles in the field, and how do we address them to fulfill its true potential?"

\section{Importance of diversity of research topics in JCR}

The JCR aims to publish manuscripts that impact the general field of drug delivery. Regardless of its specifics, it is imperative that a novel drug delivery technology provides a tangible benefit over the corresponding current standard of care. We encourage JCR authors to embrace this principle and demonstrate it experimentally in their manuscripts regardless of their technology. The drug delivery field is much bigger than any one research area. The future of the drug delivery field depends on our collective ability to identify key fundamental and practical issues, and address them through careful, self-critical studies. As we move forward, we encourage authors to join us in our mission of steering the drug delivery field into a direction that maximizes its impact on humanity. The research in drug delivery should include all aspects of formulations, ranging from pre-formulation studies and formulation design to product optimization.

The JCR also welcomes manuscripts toward applications beyond medicine. The principles and systems of controlled release are widely used in agriculture, environmental protection, food and cosmetic industries and their products, and consumer goods. The JCR provides a forum for all scientists to share their findings in diverse topics representing the entirety of delivery science and technology.

Samir Mitragotri

Twan Lammers

You Han Bae

Steven Schwendeman

Stefaan De Smedt

Jean-Christophe Leroux

Dan Peer

Ick Chan Kwon

Hideyoshi Harashima

Akihiko Kikuchi

Yu-Kyoung Oh

Vladmir Torchilin

Wim Hennink

Justin Hanes

Kinam Park 